\section{Genetic matters of CYP2D6 in breast cancer: copy number variations and nucleotide polymorphisms}

\author{
Ke-Da Yu and Zhi-Ming Shao
}

We read with great interest the content-rich Review CYP2D6 and tamoxifen: DNA matters in breast cancer (Nature Rev. Cancer 9, 576-586 (2009)) by Hoskins et al. ${ }^{1}$, which presents the current knowledge on CYP2D6 and tamoxifen in breast cancer. It is thought that CYP2D6 polymorphisms affect CYP2D6 enzyme activity, which further influences the metabolism of tamoxifen as well as the outcome of tamoxifen therapy. The authors concluded that genotype-guided tamoxifen administration might be useful as part of a strategy to optimize the treatment of breast cancer. However, there are two important questions that relate to the CYP2D6 genotype in current research. First, as the authors critically discussed, most studies have not genotyped other non-synonymous alleles except for the CYP2D6 ${ }^{*} 4$ allele in the polymorphic CYP2D6 gene ${ }^{2-4}$. Second, CYP2D6 copy number variations (CNVs) have not been fully considered. In this correspondence, we wish to draw attention to this important area of research on CYP2D6.
Genomic variability is present in many forms, including single nucleotide polymorphisms (SNPs), variable numbers of tandem repeats, copy number alterations and so on ${ }^{5}$. SNPs were previously thought to be the predominant form of genomic variation and to account for most phenotypic variation. More recently, CNVs have been identified and investigated in normal individuals. CYP2D6 is known to be an important tamoxifenmetabolizing gene that is subject to genetic deletion, duplication and multiplication, resulting in varying enzyme activity ${ }^{6}$. Here, we define 'nucleotide polymorphism' as a term representing genetic variation that includes insertion, deletion and replacement of one nucleotide or several nucleotides in CYP2D6. CNV represents a copy number change involving a DNA fragment $\geq 1 \mathrm{~kb}$. Although the understanding of CNVs is still evolving, it is acknowledged that CNVs result in differential gene expression and account for a substantial proportion of phenotypic variation. For CYP2D6, three or four or more copies have been identified ${ }^{7}$ (TABLE 1). Moreover, CYP2D6 CNV occurs

\begin{tabular}{|c|c|c|c|c|}
\hline \multirow[t]{2}{*}{ Alleles } & \multicolumn{4}{|c|}{ Copy number } \\
\hline & Null & One & Duplicated & Multiplicated $(\geq 3)$ \\
\hline Null* & 0 & 1 & 2 & $n(\geq 3)$ \\
\hline One & 1 & 2 & 3 & $n(\geq 4)$ \\
\hline Duplicated & 2 & 3 & 4 & $n(\geq 5)$ \\
\hline Multiplicated $(\geq 3)$ & $n(\geq 3)$ & $n(\geq 4)$ & $n(\geq 5)$ & $n(\geq 6)$ \\
\hline
\end{tabular}

*Null denotes an allele of CYP2D6 gene deletion (also known as CYP2D6*5).

Table 2 | The frequency of CYP2D6 gene copy number variations

\begin{tabular}{lllll|}
\hline \multirow{2}{*}{$\begin{array}{l}\text { Alleles } \\
\text { Caucasians }\end{array}$} & Asians & $\begin{array}{l}\text { Ffrican } \\
\text { Americans }\end{array}$ & $\begin{array}{l}\text { Ethiopians and } \\
\text { Saudi Arabians }\end{array}$ \\
\cline { 2 - 3 } & $2-8$ & $4-6$ & $6-7$ & $0-3$ \\
\hline One & $\sim 85$ & $\sim 95$ & $\sim 90$ & $\sim 80$ \\
$\begin{array}{l}\text { Duplicated }{ }^{\ddagger} \text { and } \\
\text { multiplicated }(\geq 3)\end{array}$ & $1-7$ & $0-1$ & 2 & $10-14$ \\
*Null denotes an allele of CYP2D6 gene deletion (also known as CYP2D6*5). ${ }^{*}$ The frequency is estimated \\
for duplication and multiplication together. Data from REFS 7,12.
\end{tabular}

at a high frequency in some populations (TABLE 2), indicating that the effect of CNVs on CYP2D6 expression is unlikely to be negligible in these populations. Therefore, we propose that, like genotyping CYP2D6 nucleotide polymorphisms, the determination of CNVs should be evaluated, at least in some populations.

Although many techniques are now available for SNP genotyping, most of them fail to determine CNVs. A reliable and high-throughput test that can identify large-scale gene multiplication or deletion is still unavailable ${ }^{6}$. However, assays such as TaqMan real-time PCR have been developed $^{8,9}$, and we encourage investigators to examine CNVs of CYP2D6 whenever possible, as the accurate determination of CNVs would warrant true associations between genotypes and phenotypes. For instance, the enzyme CYP2D $6^{\star} 1{ }^{*} 41\left({ }^{*} 41\right.$ is a common variant associated with decreased enzyme activity ${ }^{10,11}$ and is multiplied in some populations ${ }^{1,7}$ ) is classified as a poor metabolizer. However, it is difficult to determine whether $\mathrm{a}^{\star} 41$ multiplication (for example, CYP2D $6^{*} 1{ }^{*} 41 \times n$, where $n \geq 3$ ) still corresponds to poor activity. Analogously, the description of "multiplication of reduced activity alleles" in table 3 of the Review by Hoskins et al. is controversial ${ }^{1}$.

We absolutely agree with the authors' statement that large, well-designed clinical trials would support genotype-guided tamoxifen administration. However, ideally one should adopt a genotyping strategy that can decrease, to the greatest extent, genotyping bias. The assessment of CNVs should be carried out in populations with a higher incidence of CNVs in particular, and individuals who are carriers of CYP2D6 multiplication with an unknown level of function should be excluded from clinical trials.

Ke-Da Yu and Zhi-Ming Shao are at the Breast Cancer
Institute, Cancer Hospital, Shanghai Medical College,
Fudan University, Shanghai 200032,P.R. China.
Correspondence to Z.-M.S.
e-mail: zhimingshao@yahoo.com
doi:10.38/nrc2683-c1

Hoskins, J. M., Carey, L. A. \& McLeod, H. L. CYP2D6 and tamoxifen: DNA matters in breast cancer. Nature Rev. Cancer 9, 576-586 (2009).

2. Schroth, W. et al. Breast cancer treatment outcome with adjuvant tamoxifen relative to patient CYP2D6 and CYP2C19 genotypes. J. Clin. Oncol. 25 , 5187-5193 (2007).

3. Goetz, M. P. et al. The impact of cytochrome P450 2D6 metabolism in women receiving adjuvant tamoxifen. Breast Cancer Res. Treat. 101, 113-121 (2007). 4. Gjerde, J. et al. Effects of CYP2D6 and SULT1A1 genotypes including SULT1A1 gene copy number on tamoxifen metabolism. Ann. Oncol. 19, 56-61 (2008). Freeman, J. L. et al. Copy number variation: new insights in genome diversity. Genome Res. 16, 949-961 (2006). 
CORRESPONDENCE

6. Meijerman, I., Sanderson, L. M., Smits, P. H., Beijnen J. H. $\&$ Schellens, J. H. Pharmacogenetic screening of the gene deletion and duplications of CYP2D6. Drug Metab. Rev. 39, 45-60 (2007).

7. Ingelman-Sundberg, M. Genetic polymorphisms of cytochrome P450 2D6 (CYP2D6): clinical consequences, evolutionary aspects and functional diversity. Pharmacogenomics J. 5, 6-13 (2005).

8. Bodin, L., Beaune, P. H. \& Loriot, M. A

Determination of cytochrome P450 2D6 (CYP2D6) gene copy number by real-time quantitative PCR. J. Biomed. Biotechnol. 2005 248-253 (2005).

9. Schaeffeler, E., Schwab, M., Eichelbaum, M. \& Zanger, U. M. CYP2D6 genotyping strategy based on gene copy number determination by TaqMan real-time PCR. Hum. Mutat. 22, 476-85 (2003).

10. Toscano, C. et al. Impaired expression of CYP2D6 in intermediate metabolizers carrying the *41 allele caused by the intronic SNP 2988G > A: evidence for modulation of splicing events. Pharmacogenet. Genomics 16, 755-766 (2006).

11. Rau, T., Diepenbruck, S., Diepenbruck, I. \& Eschenhagen, T. The $2988 \mathrm{G}>$ A polymorphism affects splicing of a CYP2982D6 minigene. Clin. Pharmacol. Ther. 80, 555-558; author reply 558560 (2006).

12. Bradford, L. D. CYP2D6 allele frequency in European Caucasians, Asians, Africans and their descendants. Pharmacogenomics 3, 229-243 (2002). 